\title{
Development of a nomogram model predicting current bone scan positivity in patients treated with androgen-deprivation therapy for prostate cancer
}

\author{
Geoffrey T. Gotto ${ }^{1}$, Changhong Yu ${ }^{2}$, Melanie Bernstein ${ }^{3}$, James A. Eastham ${ }^{3}$ and Michael W. Kattan ${ }^{*}$ \\ 1 Southern Alberta Institute of Urology, University of Calgary, Calgary, AB, Canada \\ ${ }^{2}$ Department of Quantitative Health Sciences, Cleveland Clinic Foundation, Cleveland, OH, USA \\ ${ }^{3}$ Urology Service, Department of Surgery, Memorial Sloan Kettering Cancer Center, New York, NY, USA
}

\section{Edited by:}

Camillo Porta, Fondazione IRCCS

Policlinico San Matteo, Italy

\section{Reviewed by:}

Alan Dal Pra, Bern University Hospital, Switzerland

Camillo Porta, Fondazione IRCCS

Policlinico San Matteo, Italy

\section{*Correspondence:}

Michael W. Kattan, Department of Quantitative Health Sciences, Cleveland Clinic, Cleveland Clinic Lerner College of Medicine of Case Western Reserve University, 9500 Euclid/JJN3-01, Cleveland, OH 44195, USA

e-mail:kattanm@ccf.org

\begin{abstract}
Purpose: To develop a nomogram predictive of current bone scan positivity in patients receiving androgen-deprivation therapy (ADT) for advanced prostate cancer; to augment clinical judgment and highlight patients in need of additional imaging investigations.
\end{abstract}

\begin{abstract}
Materials and methods: A retrospective chart review of bone scan records (conventional 99mTc-scintigraphy) of 1,293 patients who received ADT at the Memorial Sloan-Kettering Cancer Center from 2000 to 2011. Multivariable logistic regression analysis was used to identify variables suitable for inclusion in the nomogram. The probability of current bone scan positivity was determined using these variables and the predictive accuracy of the nomogram was quantified by concordance index.
\end{abstract}

Results: In total, 2,681 bone scan records were analyzed and 636 patients had a positive result. Overall, the median pre-scan prostate-specific antigen (PSA) level was $2.4 \mathrm{ng} / \mathrm{ml}$; median PSA doubling time (PSADT) was 5.8 months. At the time of a positive scan, median PSA level was $8.2 \mathrm{ng} / \mathrm{ml} ; 53 \%$ of patients had PSA $<10 \mathrm{ng} / \mathrm{ml}$; median PSADT was 4.0 months. Five variables were included in the nomogram: number of previous negative bone scans after initiating ADT, PSA level, Gleason grade sum, and history of radical prostatectomy and radiotherapy. A concordance index value of 0.721 was calculated for the nomogram. This was a retrospective study based on limited data in patients treated in a large cancer center who underwent conventional ${ }^{99 \mathrm{mT}} \mathrm{c}$ bone scans, which themselves have inherent limitations.

Conclusion: This is the first nomogram to predict current bone scan positivity in ADT-treated prostate cancer patients, providing high predictive accuracy.

Keywords: non-steroidal anti-androgens, radionuclide imaging, nomogram, prostatic neoplasms, androgendeprivation therapy, bone scan positivity

\section{INTRODUCTION}

Between 15 and $40 \%$ of patients treated for localized prostate cancer will experience biochemical recurrence $(\mathrm{BCR})$, as shown by rising prostate-specific antigen (PSA) levels (1). Androgendeprivation therapy (ADT) is an accepted standard of care for patients who have received therapy with curative intent and subsequently show systemic relapse (2). Castration-resistant prostate cancer (CRPC) is defined as disease progression, typically identified by rising PSA levels and/or worsening disease according to imaging, despite castration levels of testosterone; most patients with CRPC will eventually develop metastatic (M1) disease (3).

The axial skeleton is the most common site of systemic metastasis in patients with prostate cancer (4). Recent prospective studies

Abbreviations: ADT, androgen-deprivation therapy; BCR, biochemical recurrence; CRPC, castration-resistant prostate cancer; M0, non-metastatic; M1, metastatic; PET, positron emission tomography; PSA, prostate-specific antigen; PSADT, PSA doubling time. demonstrated a median time of approximately 25-30 months from CRPC to M1 CRPC $(3,5,6)$. M1 bone disease is associated with significant morbidity, including pain, impaired mobility, and pathological fractures (7). Bone imaging can provide important information on the clinical status of asymptomatic patients with rising PSA levels. In addition, novel therapies, some of which provide benefits for patients with asymptomatic M1 CRPC, have recently been developed and approved $(8,9)$.

Although higher PSA levels and more rapid PSA doubling times (PSADT) are associated with a shorter time to bone metastasis $(5,10)$, the National Comprehensive Cancer Network prostate cancer guidelines provide minimal guidance on when to start and how often to repeat bone scan imaging in BCR patients (11). A recent study suggests that many patients classified as having non-metastatic (M0) disease are not undergoing early imaging and detection of the transition to the M1 state. In the ENTHUSE trial, a phase III trial enrolling men with M0 CRPC, $32 \%$ of screened men actually had M1 disease (based on 
magnetic resonance imaging, computed tomography, or bone scan results) (12).

Nomograms can provide individualized, disease-specific risk estimations that aid clinical management decisions (13). However, no nomogram is currently available to predict bone scan positivity in patients receiving ADT, information that could prompt relevant imaging investigations. We therefore investigated disease and treatment-related factors that could be predictive of metastases in this patient population, and used these factors to develop a nomogram model. To achieve this, we reviewed the records of a large database of patients treated at a single institution over an 11-year period who underwent conventional ${ }^{99 \mathrm{~m}}$ Tc-scintigraphy.

\section{MATERIALS AND METHODS}

This retrospective chart review included patients treated at the Memorial Sloan-Kettering Cancer Center for prostate adenocarcinoma from 2000 to 2011. All patients with bone scan records who had received ADT during this period were included in the study, with the exception of patients participating in clinical trials and those who had received prior chemotherapy or estrogen therapy (which could affect the time to bone scan positivity). Data on the frequencies of visits and reasons for the bone scans being performed were not collected. Patients were followed up from the initiation of ADT until either the first positive bone scan or the last hospital visit. Patients may have received either intermittent or continuous ADT, but these data were not collected. Data on the use of calcium, vitamin D, or bisphosphonates were not collected as these were not expected to affect bone scan positivity.

A chart review of patients' records was undertaken. A scan was coded "positive" if terms referring to metastases were identified in the records, "negative" if no such terms were found, and "unknown" if records had equivocal findings. Based on clinical relevance and data availability, eight disease and treatment variables were evaluated as potential predictors of a positive ${ }^{99 \mathrm{~m}} \mathrm{Tc}$ bone scan: number of previous negative bone scans; current PSA level; PSADT; most recent Gleason grade sum (at most recent biopsy or at prostatectomy); and a history of prior radical prostatectomy, radiotherapy, brachytherapy, or cryotherapy. PSADT was calculated based on all PSA data points measured prior to each bone scan and the interval(s) between them; negative PSADT indicated a decrease in PSA level from the previous reading. The radiotherapy group included all patients who received treatment related to the prostate. Missing values were multiply imputed before conducting statistical analyses. Testosterone data were not available for all patients and were not included in the analysis.

Multivariable logistic regression analysis was used to determine which factors should be included in the nomogram model. Restricted cubic splines were applied to continuous or nominal variables with the purpose of relaxing the commonly assumed linear association between risk factors and the outcome. Variables were selected using the step-down model reduction method (14) and identified predictors were included in the final parsimonious model on which the nomogram was built. Generalized estimating equations were used to handle clustering bone scans from the same patient. The current probability of a positive bone scan was determined by the nomogram using the patient variables at the time of each bone scan, and the predictive accuracy of the model was quantified using Harrell's concordance index (C-index) (15). The $\mathrm{C}$-index is equivalent to the area under the receiver operating characteristic curve and values can range from 0.5 , which indicates no predictive discrimination, to 1.0 , which denotes a perfect separation of patients with different outcomes $(16,17)$. Bootstrapping with 1,000 resamples was utilized to correct over-fitting bias for both the model discrimination and calibration evaluations. All statistical analyses and graphics were conducted using the open-source statistical software R version 2.14.2 (R Foundation for Statistical Computing, Vienna, Austria) in combination with the additional packages Design (Frank Harrell, Vanderbilt University, Nashville, TN, USA) and Zelig (Harvard University, Cambridge, MA, USA). The statistical hypothesis test was considered to be significant if $p<0.05$.

\section{RESULTS}

A total of 1,293 patients received ADT at the Memorial SloanKettering Cancer Center between 2000 and 2011 and underwent at least one ${ }^{99 \mathrm{~m}} \mathrm{Tc}$ bone scan during the median follow-up period of 54 months (range 3.4-144 months) from ADT initiation; 869 patients had at least one scan during ADT. Of the 424 patients who had either no scan during ADT or an unknown ADT end date, 298 had a post-ADT scan with a known ADT end date. Overall, 2,681 bone scan records were analyzed and the disease characteristics at the time of each bone scan are shown in Table 1.

Of the 1,293 patients included in the study, 636 had a positive bone scan (Table 2). A median of 0 (range $0-15$ ) negative scans were conducted after the initiation of ADT but prior to obtaining a positive scan, and the median time between the last negative scan and the positive scan was 6.7 months (range $0.5-46.7$ months). Median PSA level prior to each positive bone scan was $8.2 \mathrm{ng} / \mathrm{ml}$ (range 0-4,648 ng/ml) and median PSADT was 4.0 months (range -120 to 120 months). Around half (53\%) of the patients had PSA levels $<10 \mathrm{ng} / \mathrm{ml}$ at the time of their first positive scan.

Multivariable logistic regression analysis revealed that five variables were significant predictors of having a positive bone scan: low number of previous negative bone scans after initiating ADT, high PSA levels, high Gleason grade sum, and having no history of radical prostatectomy or radiotherapy (Table 3). These variables were then incorporated into the nomogram model. Brachytherapy, cryotherapy, and PSADT were not predictive of a positive scan (data not shown) and were not included. The final nomogram model is shown in Figure 1.

A C-index value of 0.721 was calculated for the nomogram model. As shown in Figure 2, a close relationship was observed between the results achieved with the nomogram model and the actual clinical findings (ideal calibration result).

\section{DISCUSSION}

The nomogram model presented in this study is the first analytical tool developed to predict current bone scan positivity in prostate cancer patients treated with ADT. A C-index value of 0.721 was calculated for the nomogram model, indicating high predictive accuracy. This simple nomogram could therefore be used to highlight patients in the clinic who should be a high priority for bone 
Table 1 | Patient and disease characteristics at each bone scan $(n=2,681)$.

\begin{tabular}{lc}
\hline Median (range) PSA level prior to each bone scan, ng/ml & $2.4(0-4,648)$ \\
Median (range) PSADT prior to each bone scan, months ${ }^{\mathrm{a}}$ & $5.8(-120-120)$ \\
Scan result, $n$ (\%) & \\
Positive & $636(23.7)$ \\
Negative & $2,045(76.3)$ \\
Most recent Gleason grade sum, $n$ (\%) & \\
$\leq 6$ & $267(10.0)$ \\
7 & $944(35.2)$ \\
8 & $606(22.6)$ \\
9 & $787(29.4)$ \\
10 & $63(2.3)$ \\
Missing & $14(0.5)$ \\
Pre-scan radiotherapy, $n(\%)^{\mathrm{b}}$ & \\
Yes & $1,390(51.8)$ \\
No & $1,291(48.2)$ \\
Pre-scan radical prostatectomy, $n(\%)$ & \\
Yes & $872(32.5)$ \\
No & $1,809(67.5)$ \\
Pre-scan brachytherapy, $n$ (\%) & \\
Yes & $202(7.5)$ \\
No & $2,479(92.5)$ \\
Pre-scan cryotherapy, $n$ (\%) & \\
Yes & $25(0.9)$ \\
No & \\
\hline
\end{tabular}

a PSADT was calculated based on the PSA levels measured prior to each bone scan; negative PSADT values indicated a decrease in PSA levels from the previous reading. Median (range) number of PSA values used was 13 (2-99); 95.3\% of PSADT was calculated using 3 or more PSA values.

${ }^{b} T$ he radiotherapy group included all patients who received treatment related to the prostate.

scan. This could offer substantial benefits to patients, as earlier detection of the transition from M0 to M1 CRPC may allow earlier therapeutic intervention.

Current National Comprehensive Cancer Network guidelines state that a bone scan is appropriate in men with postprostatectomy BCR (i.e., patients who have not received ADT) when symptoms develop or PSA levels increase rapidly (11). However, to date, there has not been sufficient clinical evidence to provide recommendations on imaging in men treated with ADT and, at present, the transition of patients from M0 to M1 CRPC is frequently missed (12). In the absence of detailed guidance on when to initiate imaging in ADT-treated patients with prostate cancer, our nomogram therefore has important clinical applications. For example, it could reduce potential physician subjectivity, and highlight patients who should receive an additional bone scan. Indeed, there is evidence across various therapeutic areas that nomograms can be an effective tool in predicting clinical outcomes in individual patients (18-21), with one nomogram model predicting future bone scan positivity in prostate cancer patients who had not received ADT more accurately than a group of expert clinicians (C-index values 0.812 vs. 0.628) (22). There is therefore a
Table 2 | Patient and disease characteristics at the time of the first positive scan $(n=636)$.

\begin{tabular}{lc}
\hline Median (range) PSA level prior to each bone & $8.2(0-4,648)$ \\
scan, $\mathrm{ng} / \mathrm{ml}$ & \\
PSA level (ng/ml) category, $n$ (\%) & $271(42.6)$ \\
$<5$ & $68(10.7)$ \\
$5-10$ & $57(9.0)$ \\
$10-20$ & $82(12.9)$ \\
$20-50$ & $52(8.2)$ \\
$50-100$ & $106(16.7)$ \\
$\geq 100$ & $4.0(-120-120)$ \\
Median (range) PSADT prior to each bone scan, & \\
months & $0(0-15)$ \\
Median (range) number of prior negative scans & \\
after initiating ADT & $6.7(0.5-46.7)$ \\
Median (range) time since last negative bone & \\
scan, months & \\
Pre-scan radiotherapy, $n$ (\%) & $242(38.1)$ \\
Yes & $394(61.9)$ \\
No & \\
Pre-scan radical prostatectomy, $n$ (\%) & $147(23.1)$ \\
Yes & $489(76.9)$ \\
No &
\end{tabular}

Table 3 | Multivariable logistic regression analysis of the variables included in the nomogram model.

\begin{tabular}{|c|c|c|c|}
\hline Variable & Comparison & Odds ratio ${ }^{\mathrm{a}}(95 \% \mathrm{Cl})$ & $p$-value \\
\hline PSA level (ng/ml) & 13.7 vs. $0.2^{\mathrm{a}}$ & $2.47(2.07-2.93)$ & $<0.01$ \\
\hline $\begin{array}{l}\text { Number of previous } \\
\text { negative bone scans } \\
\text { after initiating ADT }\end{array}$ & 2 vs. $0^{\mathrm{a}}$ & $0.41(0.33-0.51)$ & $<0.01$ \\
\hline $\begin{array}{l}\text { Most recent Gleason } \\
\text { grade sum }\end{array}$ & 9 vs. $7^{a}$ & $1.58(1.29-1.93)$ & $<0.01$ \\
\hline $\begin{array}{l}\text { Pre-scan radical } \\
\text { prostatectomy }\end{array}$ & Yes vs. No & $0.63(0.52-0.78)$ & 0.01 \\
\hline Pre-scan radiotherapy & Yes vs. No & $0.76(0.60-0.97)$ & 0.03 \\
\hline
\end{tabular}

${ }^{a}$ For continuous variables, the third quartile (Q3) and first quartile (Q1) are shown and were compared.

strong argument to use nomograms to augment clinical judgment and prompt further imaging investigations.

During development of the nomogram, five factors were identified as predictive of current bone scan positivity: lower number of previous negative bone scans after initiating ADT, higher PSA level, higher Gleason grade sum, and having no history of radical prostatectomy or radiotherapy. This is generally in line with previous findings in prostate cancer. Higher PSA levels $(>10 \mathrm{ng} / \mathrm{ml})$ have been associated with a shorter time to first bone metastasis in CRPC $(5,10)$, as well as decreased overall survival $(10)$. Several studies have also demonstrated the association between higher Gleason scores and an increased risk of bone metastases 
Points

$\begin{array}{lllllllllll}0 & 10 & 20 & 30 & 40 & 50 & 60 & 70 & 80 & 90 & 100\end{array}$

No. previous negative bone scans

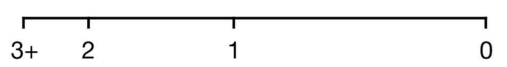

$\mathrm{PSA}(\mathrm{ng} / \mathrm{ml})$

Pre-scan radiotherapy

Pre-scan prostatectomy

Most recent Gleason grade sum

Total points

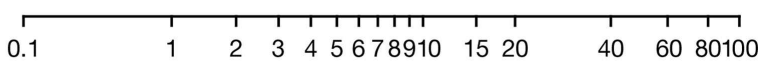

No
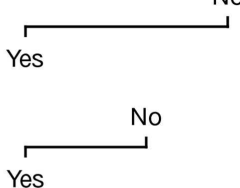

Yes
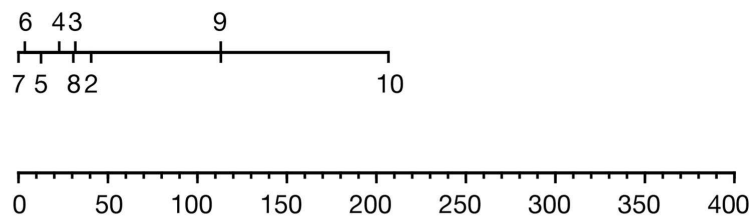

Predicted probability of having a positive bone scan
FIGURE 1 | Nomogram model developed to predict current bone scan positivity in patients treated with ADT for prostate cancer. The result for each variable has a corresponding points score (top scale). The points score for each variable is determined and summed to calculate the total points for a given patient. This value is located on the total in patients with newly diagnosed prostate cancer (23-25). Furthermore, Gleason score and current PSA levels were significant predictors of systemic progression in patients who had received previous ADT or radiotherapy (26). Although these factors do yield useful prognostic information, there is a clear need for new predictors to be identified in future studies.

Data suggest that patients who have received ADT may have a different risk of bone metastasis than those who have not, and this should be taken into account when monitoring PSA levels. In our study, PSA level at the time of a positive scan appeared to be highly variable (range $0-4,648 \mathrm{ng} / \mathrm{ml}$ ); however, the proportion of patients with PSA levels $<10 \mathrm{ng} / \mathrm{ml}$ at the time of positive bone scan was high at 53\%. A similar analysis in men who had not received ADT found that PSA levels were lower at the time of M1 diagnosis (25.9\% of patients had PSA $<10 \mathrm{ng} / \mathrm{ml}$, $50.8 \%$ had PSA $10-100 \mathrm{ng} / \mathrm{ml}$, and $23.3 \%$ had PSA $>100 \mathrm{ng} / \mathrm{ml}$ ) (27). In another study, men with BCR on ADT were significantly more likely to have a positive scan than patients who had not received ADT, when the results were adjusted for PSA level, PSADT, and PSA velocity (odds ratio 5.00; $p=0.004$ ) (28). These findings suggest that data obtained in patients who have not received ADT cannot necessarily be extrapolated to patients treated with ADT, and that rising PSA, irrespective of the absolute value, should highlight the possibility of bone metastasis in this patient population. points scale (second from the bottom). The predicted probability of having a current positive bone scan is determined by drawing a vertical line down from the total points scale to the probability scale below. Number of previous negative bone scans should only include those after the initiation of ADT.

Although the simple nomogram that we have developed could yield useful additional information to clinicians, we recognize that our study has certain limitations. Firstly, the study population was restricted to patients who underwent conventional ${ }^{99 \mathrm{~m}} \mathrm{Tc}$ bone scans, which themselves have inherent limitations. These have relatively low specificity and sensitivity for detecting bone metastases compared with other imaging techniques (29), and they are rarely able to detect soft tissue or visceral metastases. Resources did not allow us to confirm that all patients lacked a positive prior bone scan. All patients with known prior positive bone scans were excluded, and as such, this tool should not be used in a patient who has a known prior positive bone scan. Secondly, this was a retrospective study based on the limited data collected nonsystematically by different clinicians within a large cancer center. For example, complete data on the timing of the development of CRPC and bone metastases were not available, which could have provided useful diagnostic information. External validation of the nomogram is needed, and a prospective analysis with a clearly defined testing and treatment algorithm would yield additional valuable data to confirm and extend these findings. Thirdly, the patients in this study were referred for treatment at the Memorial Sloan-Kettering Cancer Center and may not be reflective of the types of patients managed in the community setting. In addition, the bone scan images were not obtained according to a specific schedule, and the timing of bone scan imaging may have been 


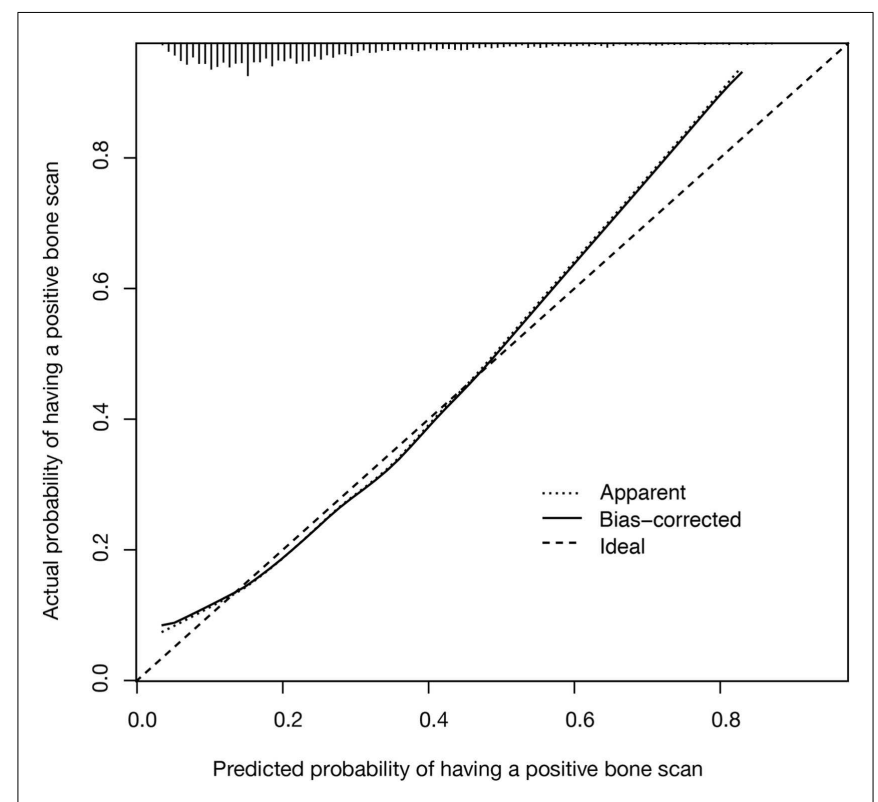

FIGURE 2 | Calibration plot for the nomogram model showing predicted vs. actual likelihood of having a positive bone scan is shown.

The vertical lines along the top of the figure show the distribution of nomogram-predicted probabilities for patients in the study cohort.

influenced by disease or treatment factors that were not evaluated in our chart review.

Avenues of further research became apparent during the course of this analysis. The use of ${ }^{99 \mathrm{~m}} \mathrm{Tc}$ bone scintigraphy as the standard first-line imaging technique is now being challenged by more sensitive and more specific modalities, such as magnetic resonance imaging and $\left[{ }^{18} \mathrm{~F}\right]$-fluoride positron emission tomography (PET) (30). Evaluation of other imaging techniques used to detect bone metastases in prostate cancer are already underway, most recently the use of $\left[{ }^{18} \mathrm{~F}\right]$ sodium fluoride PET as part of the National Oncologic PET Registry. The evidence gathered from this registry will provide information on the clinical impact of sodium fluoride PET in practice and the viability of expanding reimbursement to cover sodium fluoride imaging. Therefore, nomograms that predict the risk of metastases following the use of newer as well as existing imaging techniques could also be developed. Secondly, the discriminatory power of our nomogram, as indicated by the C-index, was 0.721 . Although this is comparatively high accuracy, there are clearly additional factors that contribute to the development of the M1 state. It is therefore important to identify these factors and to understand their interaction with the predictors identified in this analysis. Given that there are now several therapies developed for the treatment of M1 CRPC, including sipuleucel-T, abiraterone acetate, enzalutamide, and cabazitaxel, earlier detection may provide additional benefit for the patient. Finally, although our study did not define the transition to CRPC, patients who developed new bony metastases following the initiation of ADT have presumably already developed castration-resistant disease. Therefore, the disease variables identified as accurate predictors of a positive bone scan in this study may also be predictive of the transition from castration-sensitive disease to CRPC, which warrants further investigation.

\section{CONCLUSION}

In conclusion, this study describes the first nomogram model to predict current bone scan positivity with a high level of accuracy in patients with prostate cancer who have received ADT. The nomogram could be used to highlight patients in the clinic who should be a high priority for bone scan. Therefore, integrating this nomogram into the clinical decision-making process could inform decisions on imaging and allow earlier detection of metastases, which could improve the care of ADT-treated patients.

\section{AUTHOR CONTRIBUTIONS}

Geoffrey T. Gotto, Changhong Yu, Melanie Bernstein, James A. Eastham, and Michael W. Kattan received no compensation related to the development of this manuscript, are fully responsible for all content and editorial decisions, and meet criteria for authorship as recommended by the International Committee of Medical Journal Editors.

\section{ACKNOWLEDGMENTS}

Writing and editorial assistance was provided by Laura Gibbons, $\mathrm{PhD}$, and Mike Parsons, PhD, of Complete HealthVizion, UK, which was funded by Dendreon Corporation. The authors would like to acknowledge the individuals who participated in the review of patients' records: Nicole Benfante, Michael Goltzman, Sheila Mathew, Elizabeth Medina, Tanya Milan, Michael Nieves, and Jennifer Veloz. Funding/Support and role of the sponsor: the development of the manuscript was supported by Dendreon Corporation. Internal review board approval: Institutional review board (IRB) approval was required (IRB approved protocol number: WA0268-11).

\section{REFERENCES}

1. Ward JF, Moul JW. Biochemical recurrence after definitive prostate cancer therapy. Part I: defining and localizing biochemical recurrence of prostate cancer. Curr Opin Urol (2005) 15:181-6. doi:10.1097/01.mou.0000165553.17534.e3

2. Heidenreich A, Bastian PJ, Bellmunt J, Bolla M, Joniau S, van der Kwast T, et al. Guidelines on Prostate Cancer. (2014). Available from: http://www.uroweb.org/ guidelines/online-guidelines/

3. Nelson JB, Love W, Chin JL, Saad F, Schulman CC, Sleep DJ, et al. Phase 3 , randomized, controlled trial of atrasentan in patients with nonmetastatic, hormone-refractory prostate cancer. Cancer (2008) 113:2478-87. doi:10.1002/ cncr.23864

4. Coleman RE. Clinical features of metastatic bone disease and risk of skeletal morbidity. Clin Cancer Res (2006) 12:6243s-9s. doi:10.1158/1078-0432.CCR06-0931

5. Smith MR, Kabbinavar F, Saad F, Hussain A, Gittelman MC, Bilhartz DL, et al. Natural history of rising serum prostate-specific antigen in men with castrate nonmetastatic prostate cancer. J Clin Oncol (2005) 23:2918-25. doi:10.1200/ JCO.2005.01.529

6. Smith MR, Saad F, Coleman R, Shore N, Fizazi K, Tombal B, et al. Denosumab and bone-metastasis-free survival in men with castration-resistant prostate cancer: results of a phase 3, randomised, placebo-controlled trial. Lancet (2012) 379:39-46. doi:10.1016/S0140-6736(11)61226-9

7. Nemeth JA, Yousif R, Herzog M, Che M, Upadhyay J, Shekarriz B, et al. Matrix metalloproteinase activity, bone matrix turnover, and tumor cell proliferation in prostate cancer bone metastasis. J Natl Cancer Inst (2002) 94:17-25. doi:10.1093/jnci/94.1.17

8. Cheever MA, Higano CS. PROVENGE (Sipuleucel-T) in prostate cancer: the first FDA-approved therapeutic cancer vaccine. Clin Cancer Res (2011) 17:3520-6. doi:10.1158/1078-0432.CCR-10-3126 
9. Hoffman-Censits J, Kelly WK. Enzalutamide: a novel anti-androgen for patients with castrate resistant prostate cancer. Clin Cancer Res (2013) 19:1335-9. doi:10.1158/1078-0432.CCR-12-2910

10. Smith MR, Cook R, Lee KA, Nelson JB. Disease and host characteristics as predictors of time to first bone metastasis and death in men with progressive castration-resistant nonmetastatic prostate cancer. Cancer (2011) 117:2077-85. doi: $10.1002 / \mathrm{cncr} .25762$

11. NCCN Clinical Practice Guidelines in Oncology. Prostate Cancer v2. National Comprehensive Cancer Network Web site (2014). Available from: http://www. nccn.org/professionals/physician_gls/pdf/prostate.pdf

12. Yu EY, Miller K, Nelson J, Gleave M, Fizazi K, Moul JW, et al. Detection of previously unidentified metastatic disease as a leading cause of screening failure in a phase III trial of zibotentan versus placebo in patients with nonmetastatic, castration resistant prostate cancer. J Urol (2012) 188:103-9. doi:10.1016/j.juro.2012.03.008

13. Shariat SF, Karakiewicz PI, Suardi N, Kattan MW. Comparison of nomograms with other methods for predicting outcomes in prostate cancer: a critical analysis of the literature. Clin Cancer Res (2008) 14:4400-7. doi:10.1158/1078-0432. CCR-07-4713

14. Harrell FE. Regression modeling strategies with applications to linear models, logistic regression, and survival analysis. In: Per Kragh A, editor. Statistics in Medicine. New York, NY: Springer-Verlag (2001). p. 2531-2.

15. Harrell FE Jr, Califf RM, Pryor DB, Lee KL, Rosati RA. Evaluating the yield of medical tests. JAMA (1982) 247:2543-6. doi:10.1001/jama.247.18.2543

16. Lasko TA, Bhagwat JG, Zou KH, Ohno-Machado L. The use of receiver operating characteristic curves in biomedical informatics. J Biomed Inform (2005) 38:404-15. doi:10.1016/j.jbi.2005.02.008

17. Harrell FE Jr, Lee KL, Mark DB. Multivariable prognostic models: issues in developing models, evaluating assumptions and adequacy, and measuring and reducing errors. Stat Med (1996) 15:361-87. doi:10.1002/(SICI) 10970258(19960229) 15:4<361::AID-SIM168>3.0.CO;2-4

18. Ulrik CS, Løkke A, Dahl R, Dollerup J, Hansen G, Cording PH, et al. Early detection of COPD in general practice. Int J Chron Obstruct Pulmon Dis (2011) 6:123-7. doi:10.2147/COPD.S16929

19. Zaak D, Burger M, Otto W, Bastian PJ, Denzinger S, Stief CG, et al. Predicting individual outcomes after radical cystectomy: an external validation of current nomograms. BJU Int (2010) 106:342-8. doi:10.1111/j.1464-410X.2009. 09138.x

20. Nam RK, Toi A, Klotz LH, Trachtenberg J, Jewett MA, Appu S, et al. Assessing individual risk for prostate cancer. J Clin Oncol (2007) 25:3582-8. doi:10.1200/ JCO.2007.10.6450

21. Slovin SF, Wilton AS, Heller G, Scher HI. Time to detectable metastatic disease in patients with rising prostate-specific antigen values following surgery or radiation therapy. Clin Cancer Res (2005) 11:8669-73. doi:10.1158/1078-0432.CCR05- 1668

22. Kattan MW, Yu C, Stephenson AJ, Sartor O, Tombal B. Clinicians versus nomogram: predicting future technetium- $99 \mathrm{~m}$ bone scan positivity in patients with rising prostate-specific antigen after radical prostatectomy for prostate cancer. Urology (2013) 81:956-61. doi:10.1016/j.urology.2012.12.010

23. Lin K, Szabo Z, Chin BB, Civelek AC. The value of a baseline bone scan in patients with newly diagnosed prostate cancer. Clin Nucl Med (1999) 24:579-82. doi:10.1097/00003072-199908000-00007

24. Ritenour CWM, Abbott JT, Goodman M, Alazraki N, Marshall FF, Issa MM. The utilization of Gleason grade as the primary criterion for ordering nuclear bone scan in newly diagnosed prostate cancer patients. Scientific World Journal (2009) 9:1040-5. doi:10.1100/tsw.2009.113

25. Tanaka N, Fujimoto K, Shinkai T, Nakai Y, Kuwada M, Anai S, et al. Bone scan can be spared in asymptomatic prostate cancer patients with PSA of $\leq 20 \mathrm{ng} / \mathrm{ml}$ and Gleason score of $\leq 6$ at the initial stage of diagnosis. Jpn J Clin Oncol (2011) 41:1209-13. doi:10.1093/jjco/hyr118

26. Inman BA, Frank I, Boorjian SA, Akornor JW, Karnes RJ, Leibovich BC, et al. Dynamic prediction of metastases after radical prostatectomy for prostate cancer. BJU Int (2011) 108:1762-8. doi:10.1111/j.1464-410X.2011.10208.x

27. Loeb S, Makarov DV, Schaeffer EM, Humphreys EB, Walsh PC. Prostate specific antigen at the initial diagnosis of metastasis to bone in patients after radical prostatectomy. J Urol (2010) 184:157-61. doi:10.1016/j.juro.2010.03.033

28. Moreira D, Aronson W, Terris M, Presti J, Amling C, Kane C, et al. Does being on ADT alter the ability of PSA and PSADT to predict a positive bone scan? Results from the SEARCH database. J Urol (2010) 183:e335-6. doi:10.1016/j.juro.2010.02.1616

29. Even-Sapir E, Metser U, Mishani E, Lievshitz G, Lerman H, Leibovitch I. The detection of bone metastases in patients with high-risk prostate cancer: ${ }^{99 \mathrm{~m}} \mathrm{Tc}$ MDP planar bone scintigraphy, single- and multi-field-of-view SPECT, ${ }^{18} \mathrm{~F}$ fluoride PET, and ${ }^{18}$ F-fluoride PET/CT. J Nucl Med (2006) 47:287-97.

30. Tombal B, Lecouvet F. Modern detection of prostate cancer's bone metastasis: is the bone scan era over? Adv Urol (2012) 2012:893193. doi:10.1155/2012/893193

Conflict of Interest Statement: Michael W. Kattan is a consultant for GlaxoSmithKline and Merck. Geoffrey T. Gotto, Changhong Yu, Melanie Bernstein, and James A. Eastham have no financial relationships to disclose.

Received: 14 July 2014; accepted: 10 October 2014; published online: 27 October 2014. Citation: Gotto GT, Yu C, Bernstein M, Eastham JA and Kattan MW (2014) Development of a nomogram model predicting current bone scan positivity in patients treated with androgen-deprivation therapy for prostate cancer. Front. Oncol. 4:296. doi: $10.3389 /$ fonc.2014.00296

This article was submitted to Genitourinary Oncology, a section of the journal Frontiers in Oncology.

Copyright (C) 2014 Gotto, Yu, Bernstein, Eastham and Kattan. This is an open-access article distributed under the terms of the Creative Commons Attribution License (CC $B Y)$. The use, distribution or reproduction in other forums is permitted, provided the original author(s) or licensor are credited and that the original publication in this journal is cited, in accordance with accepted academic practice. No use, distribution or reproduction is permitted which does not comply with these terms. 